\title{
Intelligent Energy Management System using IoT
}

\author{
Saswati Kumari Behera, Suganya.S, S.Priyadarsini
}

\begin{abstract}
Energy management system is the most challenging job in the world. Various factors have an effect on energy management, such as financial, climate. This will limit provisional decision-making. A road map can be obtained from the energy management system for organization, efficiency, sustainability and profitability. Reducing energy losses and saving fuel are some of the energy management system's advantages. In thermal power plant, till date Auxiliary consumption value can be observed and recorded only after 24 hours. By using this project we can obtain Auxiliary consumption value in real time and unnecessary energy consumption can be precisely located and reduced. For this hardware components such as PZEM-004T, Rpi 3b+ and ESP8266 are used. NODE-RED software is used.
\end{abstract}

Keywords-auxiliary consumption, PZEM-004T sensor, ESP8266, Raspberry pi 3b+, Node-red.

\section{INTRODUCTION}

This study ' key original contribution is to devise smart techniques to reduce the auxiliary consumption cost according to standards. The energy management network is at the heart of all infrastructures, from communications, the economy and the transport of society to society. The increasing number of disruptions in the system has raised the priority of energy management system infrastructure that has been improved with technology and investment assistance; appropriate methods for optimizing the system are presented in this study.

Because the current system faces various problems caused by rising disruptions, the system operates the maximum, aging equipment, changes in load, etc. Improvement is therefore essential in order to minimize these problems. IEMS is introduced as a solution to address power problems and to prevent future failures in order to enhance the current system and solve the problems it faces. This work argues that IEMS is computational intelligence and smart meters to boost energy efficiency, stability and security. It is better, more efficient, more sustainable and more safe compared to the current system, and will reduce the number of blackouts and other failures occurring in power generation areas. Important term to be consider while discussing energy management is auxiliary consumption value, according to the norms it should be less than or equal to $8.5 \%$ of the total power generated. For which it is very important to monitor

Revised Manuscript Received on October 22, 2019

Ms.Saswati Kumari Behera, Assistant professor, Department of Electrical and Electronics Engineering, Sri Sairam Engineering college, Chennai

Suganya.S, Students, Department of Electrical and Electronics Engineering, Sri Sairam Engineering college, Chennai

S.Priyadarsini, Students, Department of Electrical and Electronics Engineering, Sri Sairam Engineering college, Chennai energy consumed by each and every feeder which are connected to various machines at various place in a power plant.

Each and every aspects of the power plant should be considered including lighting the power plant etc. So by using this project every small details of the whole power plant can be monitored in a single room, which in order reduces man power required and time consumed. And by using this method accurate instantaneous values of current, voltage and power can be obtained.

\section{LITERATURE SURVEY}

"Minimization of Auxiliary power consumption in a coal fired thermal power stations" by B.H.Narayana and M.Siddhartha Bhatt involves in changing the structure and design of pumps \& fans of motors, Hammer mills, crushers, Ball mills in order to reduce auxiliary consumption. But there is no changes involved in measuring methods. Changes in design of equipment is quiet difficult and costs very high when compared to the others methods.

\section{PROBLEM FORMULATION}

Usually energy meters are installed in each and every unit which records the total energy consumed by the particular unit but we cannot obtain the energy consumed by the individual feeders in a unit. Single unit consists of 6-8 feeders in general. Values are calculated after a certain period of time and that too calculated manually. In order to reduce auxiliary power, the energy consumed by the individual feeders at every instant must be known, so that it will be easy for the operator to find out the flaws and to eliminate it. This project paves a way to obtain a instantaneous auxiliary consumption values with the help of improved technique.

\section{IMPORTANCE OF AUXILIARY POWER CONSUMPTION}

- Equipment output loss can be easily measured by power consumption.

- The power consumption of the unit or boiler is monitored as a percentage of the generation that is only a gross index. - All major drives are covered by the power consumption guarantee on the boiler.

- In a standard coal boiler performance test, power consumption should be calculated together. 


\section{FACTORS INFLUENCING ENERGY CONSUMPTION}

- Air in leakage to boiler gas enclosure

- Fouling of Boiler heating Surface -higher dry gas loss would increase power consumption

- Higher draft loss due to air ingress and ash deposition.

- Air heater leakage / Air ingress after APH's

- Deterioration of Turbine Steam Path Condition.

- Condenser pressure/ condenser cleanliness-air ingress and extent of tube pluggage.

- Steam and water leaks from drains and vents.

\section{CURRENT METHODOLOGY}

- For auxiliary power measurement, accurate energy meters of $0.25 \%$ duly calibrated accuracy are used. total energy consumption. Individual feeder consumption is not known.

- Measurement to be carried out together with the boiler output test. For changes in the operating range, measured total power could be different from the base line values.

- Wherever boiler output has deteriorated, it should be higher.

- Gross power could be the same because, due to performance degradation, some auxiliaries may do better and some may operate at worse point.

\section{PROPOSED WORK}

The ultimate objective of energy management system is minimization of energy consumption. For this energy sensors (PZEM4T) is installed in each and every feeders so that energy consumed by the individual feeders can be obtained. If the values get exceeded than the usual consumption then there is something out of control so at that time operator can identify that particular feeder and can take necessary actions. When the operator is far away from the plant, still he can get data's through blynk app with the help of Raspberry pi which is being used for communication purpose.

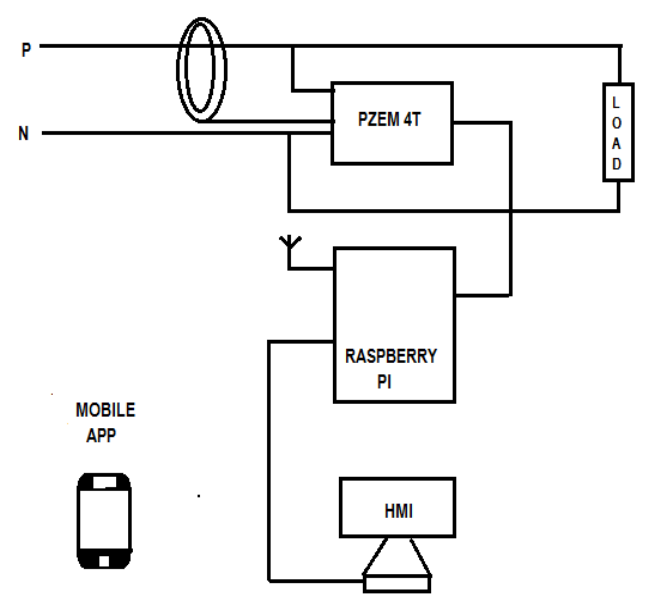

Fig (1) CIRCUIT DIAGRAM

The above circuit diagram is only for a single feeder. By following this method we can get all the data instantaneously and it requires very less time. There is no
- It covers the particular unit (6-8 feeders). This gives the

need of manual calculation. The most important thing is that energy consumed by individual feeder can be obtained which is the major advantage of this method. For this all we need is PZEM-004T sensor, ESP8266, raspberry pi, blynk application and node red software. Raspberry pi is act as a sever used for communication purpose.

\section{HARDWARE RESULTS}

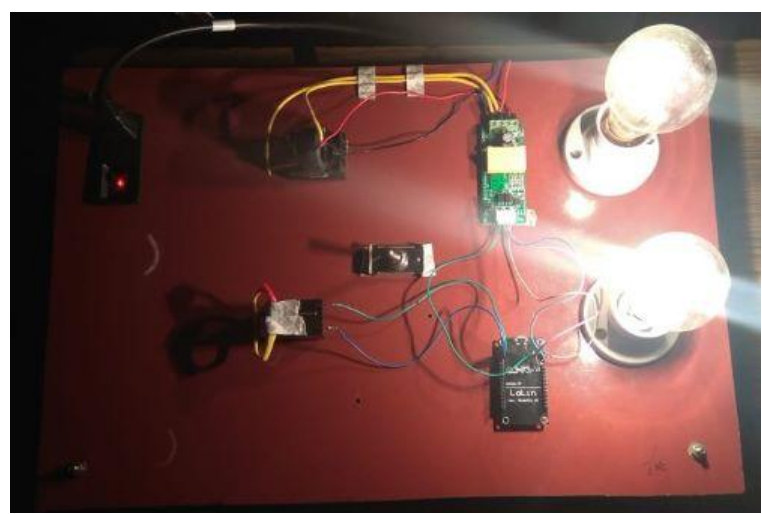

Fig(2) HARDWARE

In this model we consider two lamps as two feeders, the supply is given to lamp 1, lamp 2 and to PZEM004T sensor through current transformer. The output of the sensor is given to the ESP8266 sensor as input, the output of sensor consists data such as instantaneous power, current and voltage. The ESP8266 is programmed in such a way that it sends information to the raspberry pi through cloud.

\section{SOFTWARE RESULTS}

Software results obtained from node-red consists of three output windows. Let us discuss them briefly.

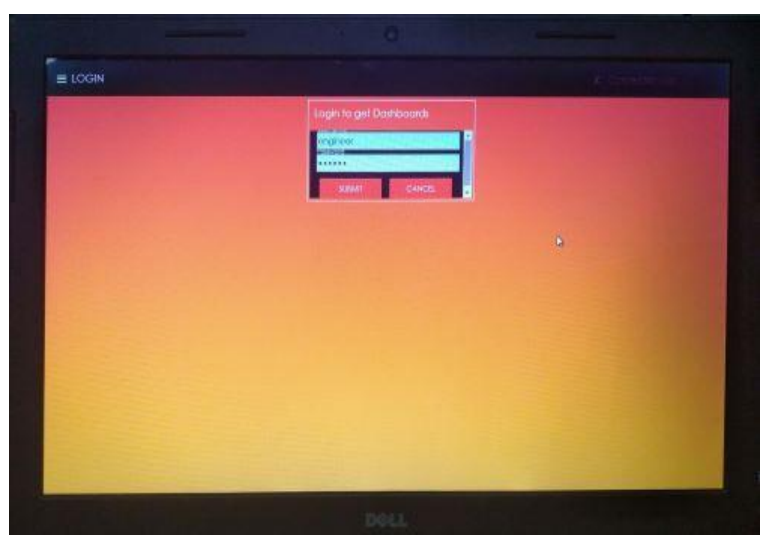

Fig(3) LOGIN SCREEN

This screen is the first screen of node-red software which comprises of login and password option. Until we enter the correct password it will not allow us to access the information.

The login id is engineer and password is pass me. For safer side we have not programmed to crash the software even after several wrong attempts to login. But if we want we can also program like that. Therefore by using node-red software we can be able to avoid usage of third person 


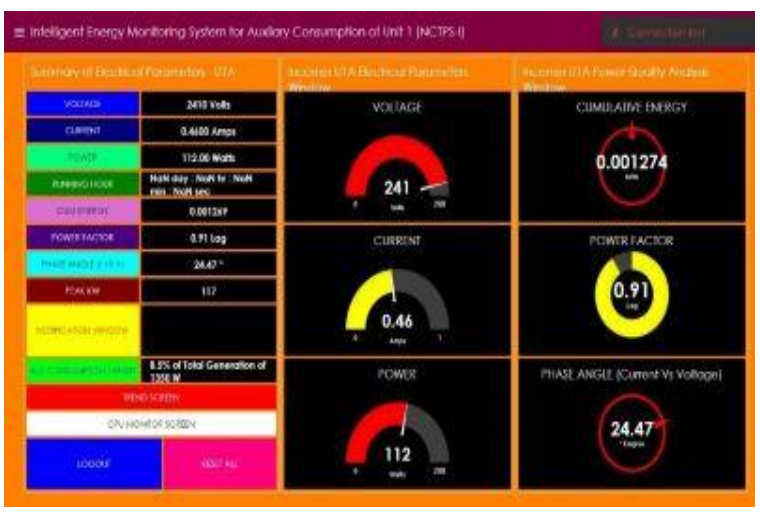

Fig(4) MAIN SCREEN 1

Figure (4) displays some of the electrical parameters such as voltage, current, power, running hour, cumulative energy,power factor,phase angle,peak $\mathrm{kW}$. These values are represented both in numerics and gauge. Apart from this there also an option to change the screen, to logout the screen and to reset all values. By clicking on the get data option we will be able to get all the data at any instant of time, which is the ultimate aim of our project.

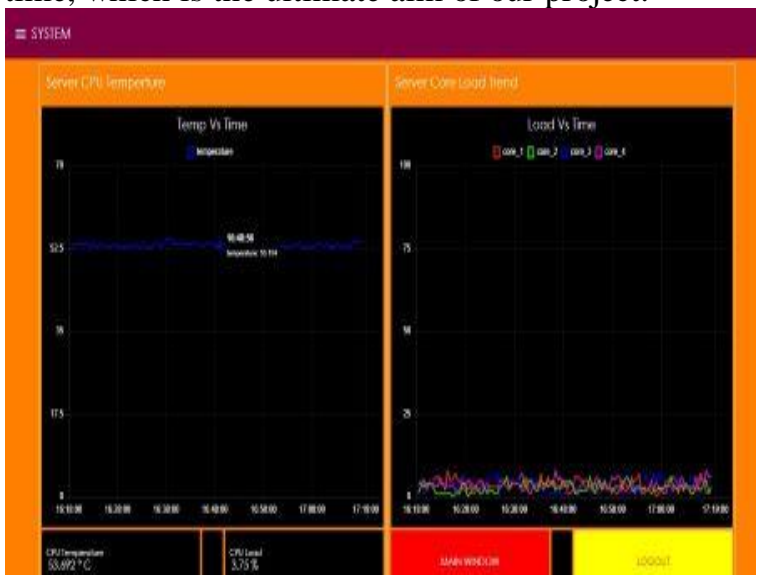

Fig(5) MAIN SCREEN 2

Figure(5) consists of two graphical representation. First graph is drawn between temperature and time.The maximum temperature that the raspberry pi can withstand is 90. This graph shows the temperature of raspberry pi instantaneously. If the value exceeds above the limit,there is a chance for malfunctioning.so that could be avoided with the help of this value and necessary actions could be taken. Second graph is drawn between load and time. This screen also contains an option to change the screen and to logout the screen.

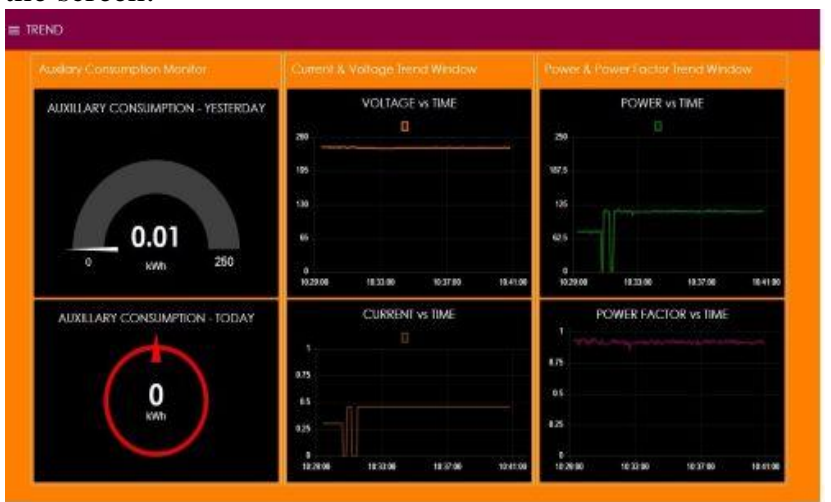

Fig(6) TREND SCREEN
Figure (6) displays the total auxiliary power consumption value for today and yesterday.then it consists of four graphical representation such as voltage vs time, current vs time, power vs time, power factor vs time.

Apart from this there is we can also get datas through a mobile application (blynk app).

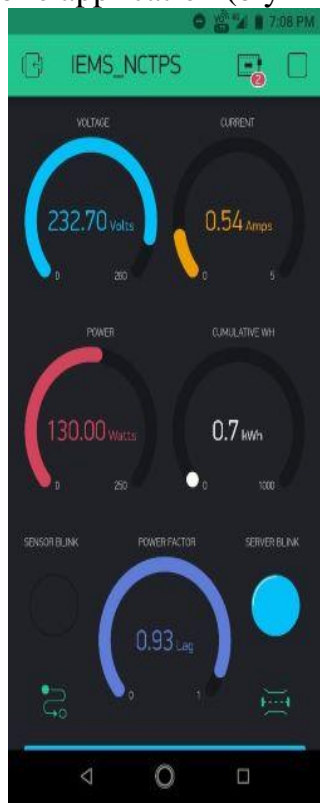

Fig (7)

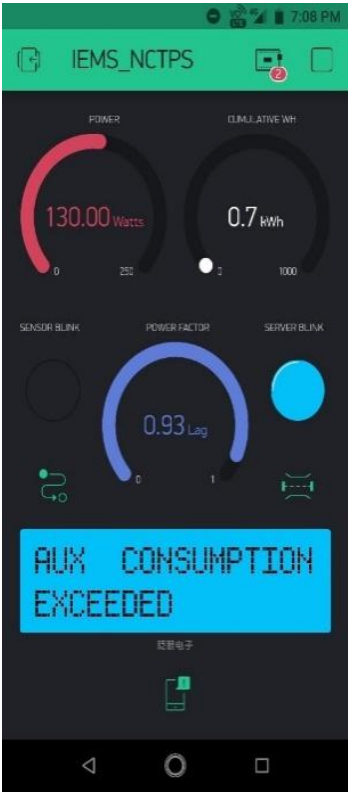

Fig(7.1)
This figure(7) comprises measurement of current, power, voltage ,power factor. When compared to node-red it has two additional options, they are using blynk application we can find whether the sensor is connected or not and it provides us SMS option which is the major advantage

Figure(7.1) represents the mobile application when the auxiliary consumption is exceeded the limit. When it exceeds the limit it gives us notification as in the diagram.

\section{CONCLUSION}

The auxiliary power efficiencies has been limited to $8.5 \%$ by the government. Normally it exceeds the limit due to some deviations. The causes of the deviation are high coal ash, excessive steam flows, internal leakage / ingress, unreliable drives, reduced quality of fuel, ageing, etc. Drawn beyond the limited value, the auxiliary power is attributed to poor coal content and its indirect effects.

Auxiliary power efficiency can be enhanced by the replacement of energy meter by power sensor(PZEM-004T) in each and every feeder. Specific energy consumption can be reduced by improving technology. As it is fully automatic method hence reduces manpower. A total approach involving operational optimization and modernization can limit the efficiencies of the AP to a value.After implementing IEMS project there is an improvement in the performance parameters i.e, plant auxiliaries effeciency,electrical energy consumption,plant performanceand fuel energy consumption. This helps in saving energy, minimize the cost of power generation so as to maximize the profit. 


\section{REFERENCES}

[1]. Young-sung son, Topipulkkinen ,Kyeong -deok moon ,chaekyukim "Home energy management system" IEEE transactions on consumer electronics, 2010.

[2]. Giovanna oriti ,AlexandrerL.Julian,NathanJ.peck "Electronics based energy management system with storage" IEEE transaction on power electronics, 2016.

[3]. Maurizio cirrincione ,MassinnoCossentio, Salvatore gaglio ,Vincent Hilare "Intelligent energy management system" IEEE explore 2009.

[4]. Bilal mubdir ,Asaad Al-Hindawi ,Noor Hadi "Design of smart energy management system for solving energy",2016.

[5]. Aswani C. \&Rathan N. (2014). "Home Energy Management System for High Power Intensive Loads", Emerging Trends in Electrical, Electronics \& Instrumentation Engineering: An International Journal (EEIEJ), Vol. 1, No. 2.

[6]. ElectricityDemand, Online Energy Resources, Online Electropedia, Woodbank Communications Ltd, UK.

[7]. H. C. Jo, S. Kim and S. K. Joo (2013). "Smart heating and air conditioning scheduling methoincorporating customer convenience for home energy management system," in IEEE Transactions on Consumer Electronics, vol. 59, no. 2, pp. 316-322.

[8]. H. V. Dange and V. K. Gondi (2011). "Powerline Communication Based Home Automation and Electricity Distribution System," International Conference on Process Automation, Control and Computing (PACC), Coimbatore, pp. 1-6.

[9]. Invernizzi, S.; Capasso, W.; Lamedica, A.; Prudenzi, R. Probabilistic Processing of Survey Collected Data in Residential Load Data for Hourly Demand Profile Extimation. In Proceedings of IEEE/TUA Athens Power Conference: "Planning, operation and control of Today's Electric Power Systems", Athens, Greece, 5-8 September 1993; pp. 866-870.

[10]. European Commission. European SmartGrids Technology Platform Vision and Strategy for Europe's Electricity Networks of the Future; Technical Report; European Commission: Louxembourg, 2006.

[11]. Capasso, W.; Grattieri, R.; Lamedica, A.; Prudenzi, R. A bottom-up approach to residential load modeling. IEEE Trans. Power Syst. 1994, 9, 957-965.

[12]. Rajan, C. Demand Side Management Using Expert System. In Proceedings of the Conference on Convergent Technologies for Asia-Pacific Region TENCON 2003, Bangalore, India, 15-17 October 2003.

[13]. B.H.Narayana and M.Siddhartha Bhatt, Minimization of Auxiliary power consumption in coal fired thermal power plants", national conference. Load Management," Wikipedia [Online] Available: http://en.wikipedia.org/wiki/Load_management [Apr. 28, 2014]. 